\section{Research Square \\ Preprints are preliminary reports that have not undergone peer review. \\ They should not be considered conclusive, used to inform clinical practice, or referenced by the media as validated information.}

\title{
Transcriptome reveals insights into biosynthesis of ginseng polysaccharides
}

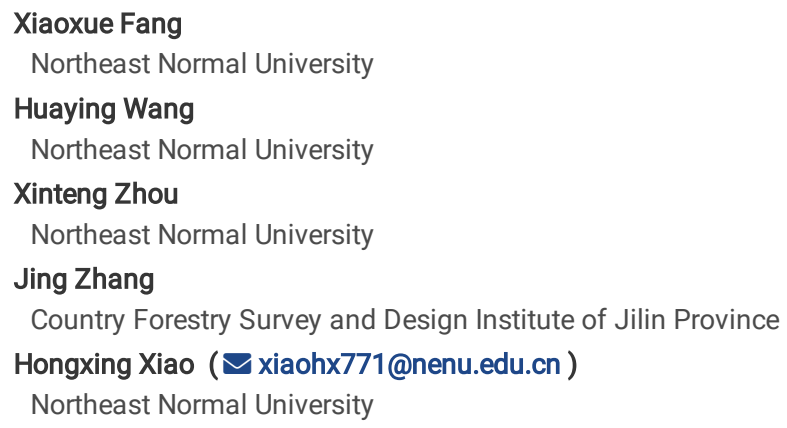

\section{Research Article}

Keywords: ginseng, ginseng polysaccharide, differentially expressed genes, transcriptomes, transcription factors

Posted Date: February 22nd, 2022

DOI: https://doi.org/10.21203/rs.3.rs-1357165/v1

License: (c) (i) This work is licensed under a Creative Commons Attribution 4.0 International License. Read Full License 


\section{Abstract}

Background: Ginseng polysaccharides, as an important active ingredient, has been used to treat various diseases. Nevertheless, the biosynthesis of ginseng polysaccharides is poorly understood. To elucidate the biosynthesis mechanism of ginseng polysaccharides, the transcriptome analysis and combined polysaccharide content determination were performed on the roots, stems and leaves collected from four cultivars of ginseng.

Results: The results indicated that the total contents of nine monosaccharides were highest in the roots. Moreover, the total content of nine monosaccharides in the roots of the four ginseng cultivars were different, but were similar in stems and leaves. Glucose was the most component of all monosaccharides. In total, 19 potential enzymes for the synthesis of ginseng polysaccharides were identified, which 17 enzymes were significantly associated with polysaccharides content. Among these genes, phosphoglucomutase (PGM), glucose-6-phosphate isomerase (GPI), UTP--glucose-1-phosphate uridylyltransferase (UGP2), fructokinase (scrK), mannose-1-phosphate guanylyltransferase (GMPP), phosphomannomutase (PMM), UDP-glucose 4-epimerase (GALE), beta-fructofuranosidase (sacA) and sucrose synthase (SUS) were correlated with MYB, AP2/ERF, bZIP and NAC transcription factors. These transcription factors may regulate the expression of genes involved in ginseng polysaccharides synthesis.

Conclusions: Our findings could provide an insight for a better understanding of the regulatory mechanism of polysaccharides biosynthesis, and would drive progress in genetic improvement and plantation development of ginseng.

\section{Background}

Panax ginseng A.C. Meyer, which belongs to the Araliaceae, is a well-known traditional Chinese medicine, which has been used for several thousands of years. Particularly in China, Korea and Japan [1]. Ginseng produces several bioactive compounds which are beneficial to human health, including ginsenosides, polysaccharides, proteins, vitamins, alkaloids and flavonoids, etc. [2-4]. Modern pharmacological researches showed that ginsenoside was most effective component. In recent years, research on ginsenosides mainly focused on the pharmacological mechanism of ginseng [5]. Whereas ginsenosides are not the only effective medicinal ingredients in ginseng. Polysaccharides have attracted more and more attention from medical scientists and nutritionists due to its important biological activity [6]. Since the middle of the 20th century, numerous studies have been conducted on the purification, structure and biological activity of ginseng polysaccharides. Modern pharmacological studies indicated ginseng polysaccharides had various biological functions, for instance hypoglycemic action, immune-enhancing activities and antioxidant activities [7, 8].

Ginseng polysaccharides could be produced by different tissues, such as roots, leaves, flowers and berries, and ginseng polysaccharides from these four different organs are varied in content, structure and biological activity [9]. Ginseng polysaccharides were biological macromolecules composed of several monosaccharide units linked by glycosidic bonds [10,11]. Previous studies have shown that the monosaccharide composition contained arabinose (Ara), rhamnose (Rha), galactose (Gal), xylose (Xyl) and glucose (Glc) et al in ginseng [12, 13]. At present, most researches on ginseng polysaccharides still focus on the pharmacological activity, however, the molecular mechanism of ginseng polysaccharide synthesis remains unrevealed.

In Chain, cultivated ginsengs are grouped into four cultivars according to the cultivation regions and morphological differences; these cultivars are GAOLI, COMMON, SHIZHU and BIANTIAO ginsengs. COMMON ginseng, SHIZHU ginseng and BIANTIAO ginseng are primarily cultivated in Fusong County of Jilin Province, the Kuandian Manchu Autonomous County of Liaoning Province and Ji'an city of Jilin Province, respectively [14]. GAOLI ginseng was introduced from the Korean Peninsula and currently was primarily cultivated in the Korean Autonomous County of Changbai and Baishan city, Jilin Province, which had developed a new cultivar in China [15]. There are great differences in root morphology, stress resistance and intrinsic quality among the four cultivars. The COMMON ginseng exhibits higher cold resistance than others cultivars [14]. In addition to the pharmacological activity of polysaccharides, previous studies have shown that increasing sugar content can reduce or avoid cold damage to plants [16]. Whether the high cold resistance of common ginseng is related to the high content of ginseng polysaccharide? And whether there are differences of polysaccharide content in four ginseng cultivars?

In our study, we integrated transcriptome sequencing and measurement of the polysaccharide content in the root, stem and leaf from four ginseng cultivars grown in China. Our specific objectives include: 1) to investigate the differences of gene expression and polysaccharides accumulation in different ginseng cultivars; 2) to infer the synthetic pathway of ginseng polysaccharides; 3 ) to explore key regulatory genes and regulatory mechanisms in the biosynthetic pathway of polysaccharides. The results of this work may improve our understanding of regulatory mechanism and provide new information to develop ginseng plantation for commercial polysaccharides production in China.

\section{Methods}

Plant Materials

Six-year-old roots, stems and leaves from four $P$. ginseng cultivars [GAOLI ginseng (GL), COMMON ginseng (CM), SHIZHU ginseng (SZ) and BIANTIAO ginseng (BT)] were used in this study. Three biological replicates were collected for each ginseng cultivar (CM had only two replicates) (Table S1). Ginseng root, stem and leaf material were harvested when the fruit was ripe. The root, stem and leaf tissues were collected from the top, middle and bottom of each sampled ginseng root, stem and leaf, respectively, and preserved in liquid nitrogen for transcriptomics analyses. The remaining root, stem and leaf on each sample were collected, dried at $55^{\circ} \mathrm{C}$ and used for polysaccharides extraction.

Extraction and determination of polysaccharides

Dry root, stem and leaf powder of ginseng $(50 \mathrm{mg})$ were used to extract ginseng polysaccharides, according to the method of Zhao et al [50]. The extracted polysaccharides powder was transferred to a $10 \mathrm{ml}$ top screw cap bottle, added $4 \mathrm{~mL}$ of trifluoroacetic acid (TFA) to the bottle (2 mol/L), then the bottle mouth was closed, followed by hydrolysis at $110^{\circ} \mathrm{C}$ for 3 hours. After cooling to room temperature, methanol was added to the bottle to remove excess TFA, and the

Page 2/12 
process was repeated five times, whereafter, $500 \mathrm{ul} \mathrm{NaOH}(0.3 \mathrm{~mol} / \mathrm{L})$ and $500 \mathrm{ul}$ methanol - 1-pheny-3-methyl-5-pyrazolone (PMP) (0.5 mol/L) were added to the bottles, and reacted in a $70^{\circ} \mathrm{C}$ water bath for $40 \mathrm{~min}$. After static cooling, added $500 \mathrm{ul} \mathrm{HCL}(0.3 \mathrm{~mol} / \mathrm{L})$. Finally, an equal volume of chloroform was added for extraction, shaken and stand still to remove the organic phase, and repeated the operation three times. The supernatant was filtered through 0.22 um membrane and analyzed by High Performance Liquid Chromatography (HPLC) (Agilent Technologies, USA). Standard monosaccharides Glc, Gal, Rha, mannose (Man), Xyl, Ara, glucuronic acid (GlcA), galacturonic acid (GalA), fucose (Fuc) were analyzed by the same experimental steps. The mobile phase was selected as $0.05 \mathrm{M}$ phosphate $(\mathrm{A})$ in water, and acetonitrile $(\mathrm{B})$, and the gradient of $8 \% \mathrm{~B}$ for 45 min were used with a flow rate of $1.0 \mathrm{~mL} / \mathrm{min}$. The detection wavelength was set to $250 \mathrm{~nm}$. The target chromatographic peaks were identified by comparing the retention time with their standards. Quantification was calculated by peak integration using the external standard method. Each sample was repeated three times. To realize the relationship of polysaccharides among samples, principal coordinate analysis (PCoA) was implemented following R package models (vegan and ape).

\section{RNA Extraction and sequencing}

Total RNA was isolated from ginseng roots, stems and leaves using the TRIzol Reagent (Invitrogen, USA) following the manufacturer's instructions, and the quantity of the RNA was assessed by NanoDrop 2000 (Thermo Scientific, USA) and gel electrophoresis. The mRNA was purified from total RNA using poly-T oligo-attached magnetic beads. After quality control (Agilent 2100 Bioanalyzer; ABI StepOnePlus Real-Time PCR System (TaqMan Probe), USA) and library preparation, cDNA was sequenced in PE150 (paired ends mode, 150 bp) using Illumina HighSeq Xten.

RNA-seq Data Analysis

The raw reads were filtered by removing adaptor sequences and low-quality sequences (Phred quality scores $<10)$ using Trimmomatic (v0.36) (Bolger et al. 2014). The high-quality reads were then mapped to the reference genome of P. ginseng "Chunpoong" (Jayakodi et al. 2018) (http://ginsengdb.snu.ac.kr) with HISAT2 software (v2.1.0) [51]. The uniquely mapped reads were retained and used for further analysis. The gene function was annotated to GO databases and KEGG databases using EggNOG (v5.0) and KAAS (v2.1), respectively [52, 53]. Transcription factors (TFs) were annotated and classified using iTAK online (v1.6) based on the Plant Transcription Factor Database (PlantTFDB v5.0) [54].

Identification of differentially expressed genes (DEGs)

Gene expression levels were estimated using RSEM (v1.3.0), and the TPM (counts per length of transcript sequence per million mapped fragments) value was used to quantify gene expression levels [55]. Pearson correlation coefficients (PCC) of expression levels were calculated between each pair of ginseng cultivars using R package (v4.2.0), and the PCC values $<0.5$ across samples were removed. DEGs were identified using DESeq2 (v1.28.1) in R software (v4.2.0) ( $\mid \log 2$ FoldChangel $>1$ and $p<0.05$ ) [56]. When log2FoldChange $>1$, DEG was considered to be up-regulated. In contrast, for log2FoldChange $<-1$, it was considered a downward adjustment. Based on gene expression, principal component analysis (PCA) was performed with R packages (prcomp), which was used to reveal the relationship among samples. To identify significantly enriched GO terms and KEGG pathways of DEGs with the entire transcriptome background, GO and KEGG enrichment analyses were performed with $p<0.05$ by clusterProfiler (v3.16.1) using R package (v4.2.0) [57].

Analysis of candidate genes involved in ginseng polysaccharide biosynthesis

Based on the composition of ginseng polysaccharides, which were composed of nine monosaccharides, including Glc, Gal, Rha, Man, GlcA, GalA and Fuc et al $[7,12]$, we determined candidate genes involved in biosynthetic pathway of ginseng polysaccharides on the base of the KEGG pathway annotation, including starch and sucrose metabolism (ko00500), fructose and mannose metabolism (ko00051), galactose metabolism (ko00052) and amino sugar and nucleotide sugar metabolism (ko00520). All potential genes were classified via BLAST (v2.7.1).

Statistical analysis

The content of nine monosaccharides were expressed as mean \pm SD. One-way anOVA (ANOVA) was used to analyze differences in content of total polysaccharides between groups and the differences in the contents of nine monosaccharides within the same individual. $P<0.05$ was considered statistically significant [58]. We examined the relationships between the genes involved in polysaccharides biosynthesis by correlation analysis and the variations of nine monosaccharides and total nine monosaccharides contents in all tissues of four ginseng cultivars. The correlation was performed using the R package, with significance levels as the p-value cutoff $(p<0.05)$.

WGCNA and visualization of gene networks

In order to analyze the regulatory mechanism of ginseng polysaccharides biosynthesis and to explore possible transcriptional factors (TFs) involved, weighted gene co-expression network analysis (WGCNA) was performed. A total of 46807 genes were used to perform WGCNA analysis in R packages (v4.2.0) [59]. Next, WGCNA network construction and module detection were performed by the "blockwiseModules" function. Modules were identified using the power $=12$, the $\operatorname{minModuleSize}=30$, and the mergeCutHeight $=0.25$, and other parameters using default settings.

To identify modules associated with the content of ginseng polysaccharides, we calculated the module eigengene by the genes of each module and correlated these eigengenes with the ginseng cultivars and tissues. Modules with $p<0.05$ and |correlation coefficients| $(r)>0.85$ were considered as significant related modules. In order to further investigate the key genes in these modules, the genes in significant related module were analyzed based on KEGG pathway annotation. Cytoscape (v3.7.0) was used to visualize the most significantly correlated genes with a WGCNA edge weight $>0.50$, then we considered the top $20 \%$ of the connected genes as hub genes in the module [60]. The correlation analysis between genes in the pathway of polysaccharides synthesis and hub genes in the module was analyzed using R package (v4.2.0).

RT-qPCR validation 
To verify the differentially expressed transcripts from RNA-Seq, we used quantitative real-time PCR assay. We selected 10 genes to validate the reliability of the transcriptome data, and these gene-specific primers were designed for ten genes by Primer Premier 6.0. In experiments for cultivars-specific expression confirmation, the relative expression levels of the candidate genes were calculated with the 2- $\triangle \Delta \mathrm{CT}$ method using GAPDH as the internal reference gene [61]. PCR amplification was performed: $95^{\circ} \mathrm{C}$ for $5 \mathrm{~min}, 40$ cycles of $95^{\circ} \mathrm{C}$ for $30 \mathrm{~s}$ and $60^{\circ} \mathrm{C}$ for $30 \mathrm{~s}$, and with a dissociation stage of $95^{\circ} \mathrm{C}$ for $15 \mathrm{~s}, 60^{\circ} \mathrm{C}$ for $60 \mathrm{~s}$ and $95^{\circ} \mathrm{C}$ for $15 \mathrm{~s}$. All the reactions in all experiments were repeated three times.

\section{Results}

Polysaccharide accumulation in different ginseng cultivars

In this study, the content of nine monosaccharides in the roots, stems and leaves of four cultivars were determined by HPLC. The total content of nine monosaccharides in roots was higher than that in stems and leaves, and that in stems and leaves were similar (Fig. 1). Among those samples, the total content of nine monosaccharides was varied. In the roots, the total content of nine monosaccharides was the highest in $\mathrm{CM}$, followed by the $\mathrm{BT}$ and $\mathrm{GL}$, and the lowest in $S Z(P<0.05$, Fig. 1A). The total content of nine monosaccharides was similar of the four cultivars in the stems and leaves $(P<0.05$, Fig. $1 A)$. $P C A$ score plot of four cultivars based on the nine monosaccharides content, and the results showed that the first two principal components (PC) explained $96 \%$ of the total variation $(P C 1=86 \%, P C 2=10 \%$ ). The all stems and leaves from four cultivars were clustered together, and clearly separated from roots, indicating significant differences in polysaccharides accumulation among tissues (Fig. 2A). In addition, Glc was the most component of monosaccharides in all samples $(\mathrm{P}<0.05$, Table 1).

Table 1

The content of nine monosaccharides in each sample.

\begin{tabular}{|c|c|c|c|c|c|c|c|c|c|c|c|c|}
\hline & & $\begin{array}{l}\mathrm{GL} \\
(\mathrm{mg} / \mathrm{g})\end{array}$ & & & $\begin{array}{l}\mathrm{CM} \\
\text { (mg/g) }\end{array}$ & & & $\begin{array}{l}\mathrm{SZ} \\
(\mathrm{mg} / \mathrm{g})\end{array}$ & & & $\begin{array}{l}\text { BT } \\
(\mathrm{mg} / \mathrm{g})\end{array}$ & \\
\hline & Root & Stem & Leaf & Root & Stem & Leaf & Root & Stem & Leaf & Root & Stem & Leaf \\
\hline Man & 0.0290 & 0.0277 & 0.0339 & 0.0358 & 0.0267 & 0.0277 & 0.0268 & 0.0271 & 0.0272 & 0.0307 & 0.0276 & 0.0288 \\
\hline GlcA & 0.0587 & 0.0508 & 0.0501 & 0.0657 & 0.0500 & 0.0498 & 0.0588 & 0.0502 & 0.0503 & 0.0681 & 0.0510 & 0.0524 \\
\hline Rha & 0.0382 & 0.0292 & 0.0292 & 0.0477 & 0.0283 & 0.0279 & 0.0340 & 0.0281 & 0.0287 & 0.0455 & 0.0288 & 0.0306 \\
\hline GalA & 0.0389 & 0.0304 & 0.0298 & 0.0497 & 0.0293 & 0.0324 & 0.0365 & 0.0294 & 0.0323 & 0.0488 & 0.0294 & 0.0304 \\
\hline Glc & $15.6215^{\star \star}$ & $1.5288^{* \star}$ & $1.3461^{\star *}$ & 17.2789 ** & $1.1827^{\star \star \star}$ & $1.5607^{\star \star *}$ & $4.2758^{\star \star}$ & $1.5825^{\star \star}$ & $1.1975^{\star \star}$ & $15.0773^{\star \star}$ & $1.3242^{\star \star \star}$ & $1.4523^{\star}$ \\
\hline Gal & 0.0513 & 0.0631 & 0.0639 & 0.0561 & 0.0537 & 0.0895 & 0.0481 & 0.0539 & 0.0722 & 0.0499 & 0.0578 & 0.0868 \\
\hline Xyl & 0.0394 & 0.0349 & 0.0337 & 0.0388 & 0.0334 & 0.0334 & 0.0361 & 0.0346 & 0.0335 & 0.0373 & 0.0346 & 0.0346 \\
\hline Ara & 0.0452 & 0.0470 & 0.0485 & 0.0475 & 0.0443 & 0.0557 & 0.0441 & 0.0450 & 0.0511 & 0.0458 & 0.0465 & 0.0591 \\
\hline Fuc & 0.0451 & 0.0448 & 0.0450 & 0.0451 & 0.0448 & 0.0452 & 0.0449 & 0.0449 & 0.0451 & 0.0452 & 0.0450 & 0.0451 \\
\hline
\end{tabular}

Note: The significance of glucose content in nine monosaccharides

*Significant at the 0.05 probability level.

**Significant at the 0.01 probability level.

Differential expression genes in four cultivars

To comprehensively investigate the differences in gene expression levels of four ginseng cultivars, we performed transcriptome sequencing for GL, CM, SZ and BT. In total, we sequenced 33 libraries from the roots, stems and leaves of four cultivar samples (Additional file 2: Table S1). For further analysis, lowquality sequences were filtered out, and $241.37 \mathrm{G}$ clean reads were obtained from the 33 libraries. Using the 'Chunpoong' genome as a reference genome, we mapped an average of $79.27 \%, 78.33 \%$ and $77.65 \%$ of clean reads for the roots, stems and leaves, respectively (Additional file 3: Table S2). The heatmaps of PCC values showed that the biological replicates had similar expression patterns and an extremely high PCC value (PCC $>0.80)$, except for BT1_R (average PCC = 0.44; Additional file 1: Fig. S1). Therefore, sample BT1_R was discarded from all subsequent analyses.

Based on the transcriptomic profile, PC1 and PC2 together explained $56 \%$ and $17 \%$ of gene expression variances among all samples, respectively. It is worth noting that the PCA score map showed stems and leaves tissue are clustered together and significant segregation from roots, indicating that gene expression at the transcriptome level responded to tissue changes, which was consistent with the results of the polysaccharides content (Fig. 2B). DEGs were identified from the samples of different tissues of each ginseng cultivars, we found that the number of DEG between GL and SZ samples was the highest in roots and stems, while the number of DEGs was the highest between CM and BT samples in leaves. The fewest DEGs were detected between BT and SZ samples in roots and leaves. In stems, there were the fewest DEGs between GL and BT (Additional file 1: Fig. S2).

Next, to better understand the functions of DEGs, we performed the KEGG enrichment analysis and GO category enrichment analysis. The DEGs of roots, stems and leaves were enriched in some secondary metabolic pathways, such as glutathione metabolism and flavonoid biosynthesis, as well as MAPK signaling pathway, protein processing in endoplasmic reticulum and plant-pathogen interaction et al (Fig. 3). In the GO enrichment analysis, the enriched terms 
of the DEGs included response to chitin, response to high light intensity, photosynthesis, and phenylalanine ammonia-lyase activity in roots, stems and leaves (Additional file 1: Figs. S3, S4, S5).

Analysis of ginseng polysaccharides biosynthetic pathway

To identify genes involved in the biosynthetic pathway of ginseng polysaccharides, we annotated the genes related to starch and sucrose metabolism (ko00500), fructose and mannose metabolism (ko00051), galactose metabolism (ko00052) and amino sugar and nucleotide sugar metabolism (ko00520). Based on the main monosaccharide components in ginseng polysaccharides, we outlined potential biosynthetic pathways for the formation of ginseng polysaccharides from sucrose. Sucrose was converted to D-fructose, then D-fructose-6phosphate (D-fructose-6p) to D-mannan-6p indirectly, and from Dmannan-1p to GDP-D-Man, subsequently, GDP-4-oxo-6-deoxy-D-Man to GDP-L-Fuc. In addition, sucrose was instantaneously transformed into UDP-GIc, UDPglcA to UDP-D-xyl, and then UDP-D-xyl into UDP-L-Ara. Moreover, UDP-Glc was converted to D-glucose-6p and then to GDP- Fuc. In addition, UDP-Gal was also directly derived from UDP-GIc, and UDP-4-keto-6-deoxy-D-Glc was converted to UDP-4-keto-Rha and UDP-Rha (Fig. 3). The components of ginseng polysaccharides included Glc, Gal, Rha, Man, Xyl, Ara, GlcA, GalA and Fuc (Fig. 4).

Relationship between gene expression and metabolite accumulation in polysaccharides biosynthesis

In biosynthetic pathway of ginseng polysaccharides, we found 102 genes encoding 19 key enzymes that control the synthesis of ginseng polysaccharides. According to structural genes extracted from polysaccharides biosynthesis pathway, the expression levels of these genes in different samples are significantly district. We found the most of the genes encoded UTP-glucose-1-phosphate uridylyltransferase (UGP2), phosphoglucomutase (PGM) and sucrose synthase (SUS) in the root of GL and CM expressed at higher levels than that of BT and SZ. The expression level of genes encoded UDP-glucose 4-epimerase (GALE) were higher in stems and leaves of GL and SZ than of CM and BT. In addition, the genes encoded mannose-6-phosphate isomerase (MPI) and GDP-mannose 4,6-dehydratase (GMDS) expressed at highest levels in the stems of SZ. Other genes in the pathway that synthesize polysaccharides, such as UDPglucuronate decarboxylase (UXS1), UDP-arabinose 4-epimeras (UXE), UDP-glucose 6-dehydrogenase (UGDH) and hexokinase (HK) et al, The Expression levels varied in different tissues across samples (Fig. 4). These results suggested that the synthesis of ginseng polysaccharides may be a pathway for multigene cooperative regulation.

Through the correlation analysis of polysaccharides synthesis related genes and content of polysaccharides, suggesting that 17 enzymes [PGM, fructokinase (scrK), beta-fructofuranosidase (sacA), UXE, UXS1, mannose-1-phosphate guanylyltransferase (GMPP), UGP2, GALE, MPI, GDP-L-fucose synthase (TSTA3), SUS, UDP-glucuronate 4-epimerase (GAE), HK, phosphomannomutase (PMM), UGDH, GDP-mannose 4,6-dehydratase (GMDS) and glucose-6-phosphate isomerase (GPI)] were obviously correlated to the content of monosaccharide content and total polysaccharides content. In addition, the expression of genes encoding scrK (Pg_S0635.5, Pg_S1306.14, Pg_S1495.1, Pg_S0588.13, Pg_S5155.1, Pg_S2241.31 and Pg_S3153.2) was positively corrected to most of monosaccharide content and polysaccharides content, which of HK (Pg_S4434.4,Pg_S3346.1,Pg_S4929.12 and Pg_S0234.21) was negatively correlated (Additional file 4: Table S3).

Co-expression modules related to the content of polysaccharides

In our study, the difference in total content of nine monosaccharides between CM and SZ was most obvious in the root, and the number of DEGs were the most of the root in CM_Vs_SZ. We screened a co-expression module by WGCNA of 49877 genes and content of nine monosaccharides, which is come from the root of $\mathrm{CM}$ and SZ. This analysis identified 12 co-expression modules, and each containing 919 to 9747 genes (Fig. 5A). Pearson correlation analysis between module eigengenes and the content of Glc, Gal, Rha, Man, Xyl, Ara, GlcA, GalA, Fuc and total content of nine monosaccharides (total), indicated the biological importance of the two modules (greenyellow and brown). The greenyellow module obviously correlated with the content of Man $(r=0.99, P=0.002)$, $\operatorname{Glc}(r=0.91, P=0.03)$ and total $(r=0.92, P=0.03)$. The brown module was highly correlated with the content of $\mathrm{Gal}(r=-0.91, P=0.03), X y l(r=-0.95, P=0.02)$ and Ara $(r=-0.98, P=0.004)$ (Fig. $5 B)$. These results suggested that the two modules obviously correlated with the content of polysaccharides accumulation in ginseng.

The KEGG annotation showed that genes in these two modules were mainly related to metabolite pathways, such as phenylpropanoid biosynthesis (ko00940), starch and sucrose metabolism (ko00500) and amino sugar and nucleotide sugar metabolism (ko00520) (Fig. 5C, D). In addition, a large number of genes for polysaccharides biosynthesis were found in these two modules, such as genes encoding PGM, GPI, scrK, UGP2, GMPP, PMM, GALE, sacA, and SUS (Table 2). 
Table 2

Genes are involved in the ginseng polysaccharide synthesis pathway in the module

Module Gene_id Gene family

\begin{tabular}{|c|c|c|}
\hline \multirow[t]{5}{*}{ greenyellow } & Pg_S2017.3 & PGM \\
\hline & Pg_S7036.4 & GPI \\
\hline & Pg_S1306.14 & scrk \\
\hline & Pg_S1124.2 & UGP2 \\
\hline & Pg_S0167.13 & GPI \\
\hline \multirow[t]{14}{*}{ brown } & Pg_S0635.5 & scrk \\
\hline & Pg_S0953.13 & GMPP \\
\hline & Pg_S4516.21 & PMM \\
\hline & Pg_S0889.28 & GALE \\
\hline & Pg_S2031.4 & sacA \\
\hline & Pg_S0897.14 & GALE \\
\hline & Pg_S1886.12 & GMPP \\
\hline & Pg_S0061.8 & sacA \\
\hline & Pg_S0219.46 & UGDH \\
\hline & Pg_S3876.17 & sacA \\
\hline & Pg_S3604.8 & GALE \\
\hline & Pg_S3338.6 & GMPP \\
\hline & Pg_S2762.11 & SUS \\
\hline & Pg_S0455.9 & GMPP \\
\hline
\end{tabular}

Note: PGM, phosphoglucomutase; GPI, glucose-6-phosphate isomerase; scrK, fructokinase; UGP2, UTP-glucose-1-phosphate uridylyltransferase; GMPP, mannose-1-phosphate guanylyltransferase; PMM, phosphomannomutase; GALE, UDP-glucose 4-epimerase; sacA, beta-fructofuranosidase; SUS, sucrose synthase.

In order to find the key regulatory TFs related to polysaccharides biosynthesis from these two modules, we constructed a gene correlation network for each module by Cytoscape. In the greenyellow module, 6 TFs were identified, GRAS (Pg_S2354.13), MADS (Pg_S4852.3), AP2/ERF (Pg_S4672.9), MYB (Pg_S1414.8 and Pg_S4889.3) and HSF (Pg_S3558.9) (Fig. 6A). It was found that these TFs highly related to GPI, PGM and UGP2 (Fig. 6C). A total of 18 genes encoding 8 TFs were found in the brown module, including MYB (Pg_S3722.2, Pg_S7293.3 and Pg_S2010.18), bZIP (Pg_S1242.23), AP2/ERF (Pg_S6406.9, Pg_S3071.2, Pg_S0253.9, Pg_S3048.23 and Pg_S4277.1), bHLH (Pg_S0724.61, Pg_S0734.14 and Pg_S0817.8), NAC (Pg_S2569.3, Pg_S1059.27 and Pg_S3248.6), MADS (Pg_S1390.1), GRAS (Pg_S0325.10) and C2H2 (Pg_S6161.2) (Fig. 6B). These TFs were highly related to all the genes that encode scrK, GMPP, PMM, GALE, sacA and SUS in the brown module, except for Pg_S3338.6 encoding GMPP (Fig. 6D). These results suggested that these TFs might regulate the expression of genes related to ginseng polysaccharides synthesis.

qRT-PCR validation

To verify the gene expression levels produced by RNA-Seq, we performed qRT-PCR analysis on ten independent samples. We selected 10 DEGs in six compared groups, and as expected, 10 DEGs exhibited similar expression tendencies. Finally, the results show that the RNA-Seq data are accurate and useful (Additional file 1: Fig. S6).

\section{Discussion}

- Effects of cultivars on polysaccharide contents

Ginseng polysaccharides are the active ingredient in ginseng, which have anti-tumor, anti-inflammation, anti-oxidation and immunomodulatory effects [1719]. In our study, we found that the total content of nine monosaccharides was significantly higher in roots than in stems and leaves. Previous studies showed that polysaccharides were produced in different tissues of ginseng, such as roots, stems, leaves, flowers and fruits, but polysaccharides were highest in roots $[18,20]$. The result of PCA also showed that the all stems and leaves from four cultivars were clustered together, but were obviously separated from the roots, suggesting accumulation of ginseng polysaccharides was different in different tissues. Moreover, the content of ginseng polysaccharides was less in stem and leaf of four cultivars. Here it is speculated that the main synthetic tissue of ginseng polysaccharides in the roots, or ginseng polysaccharides are synthesized in different tissues of ginseng and eventually transported to the roots, just like ginsenosides [21]. It is necessary to collect different tissues of ginseng at different growth stages for the determination and analysis of polysaccharides to test this hypothesis in the future.

In addition, the total content of nine monosaccharides in the root of CM was the highest. Under the condition of environmental stress and nutrient restriction, plants accumulate some secondary metabolites to improved their own defense response [22]. After cold acclimation, the content of sucrose in tomato fruit 
increased obviously [23]. COMMON ginseng grows at lower temperatures than the other three cultivars. Thus, the high polysaccharides of COMMON ginseng may be related to its high cold resistance [14]. Meanwhile, we found that the DEGs among different samples were mainly enriched in MAPK signaling pathway and plant-pathogen interaction pathways et al. Both metabolic pathways are involved in plant defense and immune systems to improve stress resistance [24, 25]. This might further indicate that ginseng polysaccharide content is related to higher resistance.

- The key genes in synthetic pathway of ginseng polysaccharides

The structural characteristics of ginseng polysaccharides have always attracted people's interest [26, 27]. Many studies on analysis of ginseng polysaccharides composition indicated that ginseng polysaccharides are composed of Glc, Gla, Ara and Man etc [13]. However, the biosynthetic pathway of ginseng polysaccharides has not been elucidated. Through the analysis of bioinformatics and monosaccharides content determination, we identified the key enzymes of ginseng polysaccharides biosynthesis. The results showed that the precursor of polysaccharides biosynthesis was sucrose. The synthetic pathway of ginseng polysaccharides is similar to that of $P$. sibiricum, Poria cocos and Hericium frutescens [28-30]. Previous studies showed that despite the striking differences in the structure of polysaccharides, the biosynthesis process of polysaccharides was conserved [30, 31]. In the synthesis pathway of ginseng polysaccharides, 19 key enzymes were identified, and the expression levels of 17 enzymes were significantly correlated with the content of polysaccharides, suggesting these enzymes are the main enzymes in the synthesis of ginseng polysaccharides.

The most of the genes encoded UGP2, PGM and SUS expressed at higher levels in the root of GL and CM than that of BT and SZ. Moreover, the polysaccharides content was significantly higher in roots of GL and SZ than that in other tissues. During the growth of transgenic Arabidopsis thaliana, overexpression of UGP gene promoted the increase of sugar content in A. thaliana [32]. Xu et al (2015) suggested the content of intracellular polysaccharide (IPS) and extracellular polysaccharide (EPS) in Ganoderma lucidum overexpressing the PGM gene were higher than those of the wild-type strain [33]. We concluded that UGP2, PGM and SUS were key enzymes of Glc synthesis in the biosynthetic pathway of ginseng polysaccharides.

ScrK is a key enzyme that acts upstream in the biosynthesis of ginseng polysaccharides. It is responsible for converting D-fructose into D-fructose 6 phosphate, and the activity of scrK effectively controls the accumulation of starch in tomato fruit [34]. HK is also the key enzyme involved in the conversion of D-fructose to D-fructose-6p, and HK played indispensable role in sugar accumulation in apple fruits [35, 36]. Scrk and HK played a role in the same branches of the ginseng polysaccharides biosynthesis pathway, but they showed the opposite pattern of correlation with polysaccharides content in this study. They might play the contrary role in biosynthesis pathway of polysaccharides. In the polysaccharides synthesis pathway of $P$. sibiricum, HK and scrK also showed converse effects [28].

- Transcription factors acting on regulation of polysaccharides content

Gene expression is influenced by a number of external and internal factors through TFs that influence them and are bind to specific regions of the target gene promoter [37, 38]. In recent years, WGCNA analysis has been frequently used to identify specific genes and TFs for the synthesis of certain metabolites in plants $[39,40]$. We identified two modules highly related to polysaccharides accumulation using WGCNA, including greenyellow and brown modules. Multiple polysaccharides biosynthesis-related genes and TFs exhibited specific interaction patterns to modulate the ginseng polysaccharides biosynthesis in these two modules.

In the greenyellow module, MYB (Pg_S1414.8 and Pg_S4889.3) and AP2/ERF (Pg_S4672.9) were highly related to genes for polysaccharides synthesis (PGM, GPI and UGP2). The MYB transcription family is one of the largest transcription families, which have been reported to play various roles in secondary metabolism of plants [41, 42]. In Arabidopsis, Chen et al (2017) found MYBS1 and MYBS2 were involved in regulating the expression of glucose-responsive genes in seeds [43]. The AP2/ERF transcription factors have two conserved amino acid sequences. It has been reported that AP2/ERF regulated the content of starch by binding to rice starch regulator1 (RSR1), a transcription factor of the rice protein family [44]. The greenyellow module obviously correlated with the content of Glc. Meanwhile, PGM, GPI and UGP2 are the main branches of Glc synthesis in the pathway of ginseng polysaccharides. Thereby, MYB and AP2/ERF might be the key TFs regulating the expression of genes that synthesize Glc (PGM, GPI and UGP2), leading to higher the content of GIc.

In the brown module, we also found the other two TFs expected MYB and AP2/ERF, including bZIP (Pg_S1242.23) and NAC (Pg_S2569.3, Pg_S1059.27 and Pg_S3248.6), which were highly related to all the genes for polysaccharides synthesis (scrK, GMPP, PMM, GALE, sacA and SUS). The bZIP identified from maize could affect the physiological and biochemical characteristics of maize endosperm, and its overexpression changed the expression of starch biosynthesis genes in endosperm [45]. The NAC family had many members and diverse functions, and had been confirmed to play the core role in starch biosynthesis in plants [46-48]. A novel transcription factor TaNAC019-A1 could directly bind to the 'ACGCAG' motif in the promoter regions of ADP-glucose pyrophosphorylase small subunit 1 region and inhibited its expression, thereby influencing starch synthesis in wheat endosperm [49]. These TFs perhaps control the synthesis of ginseng polysaccharides by regulating the genes of ginseng polysaccharides synthesis.

\section{Conclusions}

By combining transcriptome and polysaccharides content analysis of four ginseng cultivars to reveal molecular mechanism of ginseng polysaccharides. The content of nine monosaccharides was determined by HPLC, and the result showed that the total contents of nine monosaccharides were highest in the roots. Moreover, the content of nine monosaccharides in the roots of the four ginseng cultivars was different, but similar in the stems and leaves. Among monosaccharides, Glc is the most component of monosaccharides for all samples. In total, we identified 19 potential enzymes for the synthesis of ginseng polysaccharides, of which 17 enzymes were significantly associated with polysaccharides content. By WGCNA analysis, PGM, GPI, UGP2, scrK, GMPP, PMM, GALE, sacA and SUS were correlations with MYB, AP2/ERF, bZIP and NAC TFs. These TFs might regulate the genes of ginseng polysaccharides synthesis. These results revealed the biosynthetic mechanism of ginseng polysaccharides and provides a scientific basis for the research of ginseng polysaccharides. 


\section{Declarations}

\section{Acknowledgments}

We thank Wei Zhang for helping to collected the samples in this study.

\section{Authors Contributions}

X. F. and H. W. designed the experiments. X. F. and X. Z. performed most of experiments and analyzed the data. Other authors assisted in experiments and discussed the results. X. F. and H. X. wrote the manuscript.

\section{Funding}

This work was supported by National Natural Science Foundation of China (grant numbers 31770243).

\section{Availability of data and materials}

The RNA-seq data has been submitted to NCBI SRA: PRJNA762437 and PRJNA779557.

\section{Ethics approval and consent to participate}

Four ginseng cultivars in this study were collected from Korean Autonomous county of Changbai county, Jingyu county, Shizhuzi county and Taishang town of Jilin Province. The research conducted in this study neither required approval from an ethics committee, nor involved any human or animal subjects. No specific permits were required for the described field studies. The location is not privately-owned or protected in any way. We complied with the IUCN Policy Statement on Research Involving Species at Risk of Extinction and the Convention on the Trade in Endangered Species of Wild Fauna and Flora.

\section{Consent for publication}

Not applicable.

\section{Conflict of Interest}

The authors declare that they have no competing interests.

\section{References}

1. Yun T-K: Panax ginseng-a non-organ-specific cancer preventive? The Lancet Oncology 2001, 2(1):49-55.

2. Kim SK, Park JH: Trends in ginseng research in 2010. Journal of Ginseng Research 2011, 35(4):389-398.

3. Zhao N, Cheng M, Lv W, Wu Y, Liu D, Zhang X: Peptides as Potential Biomarkers for Authentication of Mountain-Cultivated Ginseng and Cultivated Ginseng of Different Ages Using UPLC-HRMS. Journal of Agricultural and Food Chemistry 2020, 68(7):2263-2275.

4. Park S-Y, Park J-H, Kim H-S, Lee C-Y, Lee H-J, Kang KS, Kim C-E: Systems-level mechanisms of action of Panax ginseng: a network pharmacological approach. Journal of Ginseng Research 2018, 42(1):98-106.

5. Kim YJ, Zhang D, Yang DC: Biosynthesis and biotechnological production of ginsenosides. Biotechnology Advances 2015, 33(6):717-735.

6. Choi KT: Botanical characteristics, pharmacological effects and medicinal components of Korean Panax ginseng C A Meyer. Acta Pharmacologica Sinica 2008, 29(9):1109-1118.

7. Tomoda M, Shimada K, Konno C, Sugiyama K, Hikino H: Partial structure of panaxan A, a hypoglycaemic glycan of Panax ginseng roots. Planta medica 1984, 50(5):436-438.

8. Luo D, Fang B: Structural identification of ginseng polysaccharides and testing of their antioxidant activities. Carbohydrate Polymers 2008, 72(3):376381.

9. Guo M, Shao S, Wang D, Zhao D, Wang M: Recent progress in polysaccharides from Panax ginseng C. A. Meyer. Food \& Function $2021,12$.

10. Loh SH, Park JY, Cho EH, Nah SY, Kang YS: Animal lectins: Potential receptors for ginseng polysaccharides. Journal of Ginseng Research 2017, 41(1):19.

11. Cui L, Wang J, Huang R, Tan Y, Zhang F, Zhou Y, Sun L: Analysis of pectin from Panax ginseng flower buds and their binding activities to galectin-3. International Journal of Biological Macromolecules 2019, 128:459-467.

12. Solov'eva T, Arsenyuk LV, Ovodov Y: Some structural features of Panax ginseng C. A. Mey pectin. Carbohydrate Research 1969, 10:13-18. 
13. Tomoda M, Shimada K, Konno C, Sugiyama K, Hikino H: Partial Structure of Panaxan A, A Hypoglycaemic Glycan of Panax ginseng Roots1. Planta Med 1984, 50(05):436-438.

14. Su WL, Lv XM, sU YX: Planting styles of main types of strains of changbai mountains ginseng and its classification of commodities. J GINS RES 2008, 4:34-39.

15. Liu A, Q., Liu Y, L.: Main Ways of Korean Ginseng had Exported to China in the Ancient. Contemporary Korea 2015, 000(001):113-125.

16. Wang X, Liu Y, Han Z, Chen Y, Huai D, Kang Y, Wang Z, Yan L, Jiang H, Lei Y et al: Integrated Transcriptomics and Metabolomics Analysis Reveal Key Metabolism Pathways Contributing to Cold Tolerance in Peanut. Front Plant Sci 2021, 12(2597).

17. Li C, Tian Z-N, Cai J-P, Chen K-X, Zhang B, Feng M-Y, Shi Q-T, Li R, Qin Y, Geng J-S: Panax ginseng polysaccharide induces apoptosis by targeting Twist/AKR1C2/NF-1 pathway in human gastric cancer. Carbohydrate Polymers 2014, 102:103-109.

18. Jiao L, Li B, Wang M, Liu Z, Zhang X, Liu S: Antioxidant activities of the oligosaccharides from the roots, flowers and leaves of Panax ginseng C.A. Meyer. Carbohydrate Polymers 2014, 106(1):293-298.

19. Kim MH, Byon YY, Ko EJ, Song JY, Yun YS, Shin T, Joo HG: Immunomodulatory activity of ginsan, a polysaccharide of Panax ginseng, on dendritic cells. Korean Journal of Physiology and Pharmacology 2009, 13(3):169-173.

20. Wan JY, Huang WH, Zheng W, Park CW, Kim SH, Seo DB, Shin KS, Zeng J, Yao H, Sava-Segal C et al: Multiple Effects of Ginseng Berry Polysaccharides: Plasma Cholesterol Level Reduction and Enteric Neoplasm Prevention. American Journal of Chinese Medicine 2017, 45(6):1293-1307.

21. Kim YJ, Joo SC, Shi J, Hu C, Quan S, Hu J, Sukweenadhi J, Mohanan P, Yang DC, Zhang D: Metabolic dynamics and physiological adaptation of Panax ginseng during development. Plant Cell Reports 2018, 37(3):393-410.

22. Endara MJ, Coley PD: The resource availability hypothesis revisited: A meta-analysis. Functional Ecology 2011, 25(2):389-398.

23. Zhou J, Min D, Li Z, Fu X, Zhao X, Wang J, Zhang X, Li F, Li X: Effects of chilling acclimation and methyl jasmonate on sugar metabolism in tomato fruits during cold storage. Scientia Horticulturae 2021, 289:110495.

24. Boller T, He SY: Innate immunity in plants: An arms race between pattern recognition receptors in plants and effectors in microbial pathogens. Science 2009, 324(5928):742-743.

25. Yang T, Chaudhuri S, Yang L, Du L, Poovaiah BW: A Calcium/Calmodulin-regulated Member of the Receptor-like Kinase Family Confers Cold Tolerance in Plants*. Journal of Biological Chemistry 2010, 285(10):7119-7126.

26. Gao Q, Kiyohara H, Cyong J, Yamada H: Chemical properties and anti-complementary activities of polysaccharide fractions from roots and leaves of Panax ginseng. Planta Medica 1989, 55(1):9-12.

27. Zhang X, Yu L, Bi H, Li X, Ni W, Han H, Li N, Wang B, Zhou Y, Tai G: Total fractionation and characterization of the water-soluble polysaccharides isolated from Panax ginseng C. A. Meyer. Carbohydrate Polymers 2009, 77(3):544-552.

28. Wang S, Wang B, Hua W, Niu J, Dang K, Qiang Y, Wang Z: De Novo Assembly and Analysis of Polygonatum sibiricum Transcriptome and Identification of Genes Involved in Polysaccharide Biosynthesis. 2017, 18(9):1950.

29. Yang L, Tang J, Chen JJ, Peng AY, Wang QM, Rao LQ, Yang H, Zhang XW, Yang HZ, Zhang C et al: Transcriptome analysis of three cultivars of Poria cocos reveals genes related to the biosynthesis of polysaccharides. Journal of Asian Natural Products Research 2019, 21(5):462-475.

30. Zhang N, Tang Z, Zhang J, Li X, Yang Z, Yang C, Zhang Z, Huang Z: Comparative transcriptome analysis reveals the genetic basis underlying the biosynthesis of polysaccharides in Hericium erinaceus. Botanical Studies 2019, 60(1)

31. Ruas-Madiedo P, Hugenholtz J, Zoon P: An overview of the functionality of exopolysaccharides produced by lactic acid bacteria. International Dairy Journal 2002, 12:163-171

32. Li N, Wang L, Zhang W, Takechi K, Takano H, Lin X: Overexpression of UDP-glucose pyrophosphorylase from Larix gmelinii enhances vegetative growth in transgenic Arabidopsis thaliana. Plant Cell Reports 2014, 33(5):779-791.

33. Xu J-W, Ji S-L, Li H-J, Zhou J-S, Duan Y-Q, Dang L-Z, Mo M-H: Increased polysaccharide production and biosynthetic gene expressions in a submerged culture of Ganoderma lucidum by the overexpression of the homologous a-phosphoglucomutase gene. Bioprocess and Biosystems Engineering 2015, 38(2):399-405.

34. Petreikov M, Dai N, Granot D, Schaffer AA: Characterization of native and yeastexpressed tomato fruit fructokinase enzymes. Phytochemistry 2001, 58(6):841-847.

35. Zhu L-c, Su J, Jin Y-r, Zhao H-y, Tian X-c, Zhang C, Ma F-w, Li M-j, Ma B-q: Genome-wide identification, molecular evolution, and expression divergence of the hexokinase gene family in apple. Journal of Integrative Agriculture 2021, 20(8):2112-2125.

36. Wang H, Xin H, Guo J, Gao Y, Liu C, Dai D, Tang L: Genome-wide screening of hexokinase gene family and functional elucidation of HXK2 response to cold stress in Jatropha curcas. Molecular Biology Reports 2019, 46(2):1649-1660.

37. Hassani D, Fu X, Shen Q, Khalid M, Rose JKC, Tang K: Parallel Transcriptional Regulation of Artemisinin and Flavonoid Biosynthesis. Trends in Plant Science 2020, 25(5):466-476.

38. Mao J, Huang L, Chen M, Zeng W, Feng Z, Huang S, Liu T: Integrated Analysis of the Transcriptome and Metabolome Reveals Genes Involved in Terpenoid and Flavonoid Biosynthesis in the Loblolly Pine (Pinus taeda L.). Frontiers in Plant Science 2021, 12.

39. Guo Y, Gao C, Wang M, Fu FF, El-Kassaby YA, Wang T, Wang G: Metabolome and transcriptome analyses reveal flavonoids biosynthesis differences in Ginkgo biloba associated with environmental conditions. Industrial Crops and Products 2020, 158

40. Ding T, Zhang R, Zhang H, Zhou Z, Liu C, Wu M, Wang H, Dong H, Liu J, Yao JL et al: Identification of gene co-expression networks and key genes regulating flavonoid accumulation in apple (Malus × domestica) fruit skin. Plant Science 2020, 304:110747.

Page $9 / 12$ 
41. Dubos C, Stracke R, Grotewold E, Weisshaar B, Martin C, Lepiniec L: MYB transcription factors in Arabidopsis. Trends in Plant Science 2010, 15(10):573581.

42. Espley RV, Hellens RP, Putterill J, Stevenson DE, Kutty-Amma S, Allan AC: Red colouration in apple fruit is due to the activity of the MYB transcription factor, MdMYB10. Plant Journal 2007, 49(3):414-427.

43. Chen YS, Chao YC, Tseng TW, Huang CK, Lo PC, Lu CA: Two MYB-related transcription factors play opposite roles in sugar signaling in Arabidopsis. Plant Molecular Biology 2017, 93(3):299-311.

44. Fu F-F, Xue H-W: Coexpression Analysis Identifies Rice Starch Regulator1, a Rice AP2/EREBP Family Transcription Factor, as a Novel Rice Starch Biosynthesis Regulator Plant Physiology 2010, 154(2):927-938.

45. Dong Q, Xu Q, Kong J, Peng X, Zhou W, Chen L, Wu J, Xiang Y, Jiang H, Cheng B: Overexpression of ZmbZIP22 gene alters endosperm starch content and composition in maize and rice. Plant Science 2019, 283:407-415.

46. Mohanta T, Yadav D, Khan A, Hashem A, Tabassum B, Khan A, Abd Allah EF, Al-Harrasi A: Genomics, molecular and evolutionary perspective of NAC transcription factors. PLOS ONE 2020, 15:e0231425.

47. Zhang J, Chen J, Yi Q, Hu Y, Liu H, Liu Y, Huang Y: Novel role of ZmaNAC36 in co-expression of starch synthetic genes in maize endosperm. Plant Molecular Biology 2014, 84(3):359-369.

48. Zhang Z, Dong J, Ji C, Wu Y, Messing J: NAC-type transcription factors regulate accumulation of starch and protein in maize seeds. Proceedings of the National Academy of Sciences of the United States of America 2019, 166(23):11223-11228.

49. Liu Y, Hou J, Wang X, Li T, Majeed U, Hao C, Zhang X: The NAC transcription factor NAC019-A1 is a negative regulator of starch synthesis in wheat developing endosperm. Journal of Experimental Botany 2020, 71(19):5794-5807.

50. Zhao L, C.: Study on extraction of chemical constituents from Panax ginseng and optimization of test methods. Chinese Academy of Agricultural Sciences; 2019.

51. Kim D, Langmead B, Salzberg SL: HISAT: a fast spliced aligner with low memory requirements. Nature Methods 2015, 12(4):357-360.

52. Huerta-Cepas J, Szklarczyk D, Heller D, Hernández-Plaza A, Forslund S, Cook H, Mende D, Letunic I, Rattei T, Jensen L et al: eggNOG 5.0: a hierarchical, functionally and phylogenetically annotated orthology resource based on $\mathbf{5 0 9 0}$ organisms and $\mathbf{2 5 0 2}$ viruses. Nucleic acids research $2018,47$.

53. Moriya Y, Itoh M, Okuda S, Yoshizawa A, Kanehisa M: Moriya Y, Itoh M, Okuda S, Yoshizawa AC, Kanehisa M.. KAAS: an automatic genome annotation and pathway reconstruction server. Nucl Ac Res 35: W182-W185. Nucleic acids research 2007, 35:W182-185.

54. Zheng Y, Jiao C, Sun H, Rosli HG, Pombo MA, Zhang P, Banf M, Dai X, Martin GB, Giovannoni JJ et al: iTAK: A Program for Genome-wide Prediction and Classification of Plant Transcription Factors, Transcriptional Regulators, and Protein Kinases. Mol Plant 2016, 9(12):1667-1670.

55. Li B, Dewey CN: RSEM: accurate transcript quantification from RNA-Seq data with or without a reference genome. BMC Bioinformatics 2011, 12(1):323.

56. Love MI, Huber W, Anders S: Moderated estimation of fold change and dispersion for RNA-seq data with DESeq2. Genome Biology 2014, 15(12).

57. Yu G, Wang LG, Han Y, He QY: ClusterProfiler: An R package for comparing biological themes among gene clusters. OMICS A Journal of Integrative Biology 2012, 16(5):284-287.

58. Anderson MJ: A new method for non-parametric multivariate analysis of variance. Austral Ecology 2001, 26(1):32-46.

59. Langfelder P, Horvath S: WGCNA: An R package for weighted correlation network analysis. BMC Bioinformatics $2008,9$.

60. Shannon, Paul, Markeil, Andrew, Ozier, Owen, Baliga, Nitin, S., Research WJG: Cytoscape: A Software Environment for Integrated Models of Biomolecular Interaction Networks. 2003, 13(11):2498-2504.

61. Livak KJ, Schmittgen T: Analysis of relative gene expression data using real-time quantitative PCR and the 2-DDCt method. Methods 2001, 25:402-408.

\section{Figures}

(A)

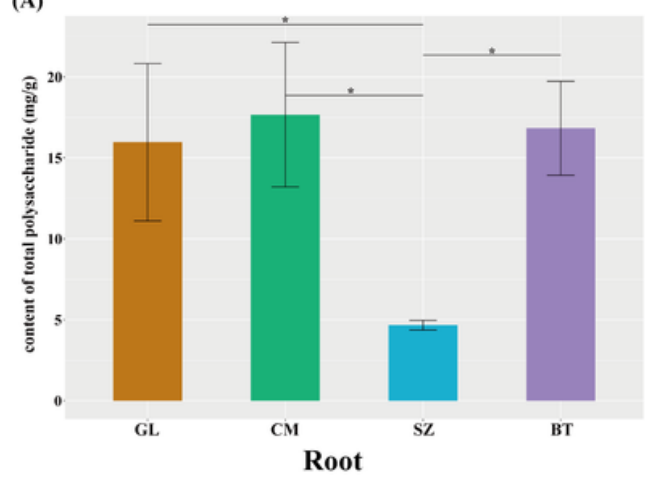

(B)

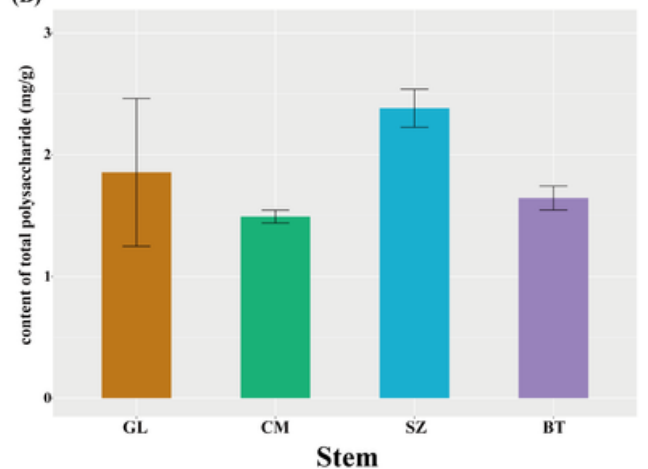

(C)

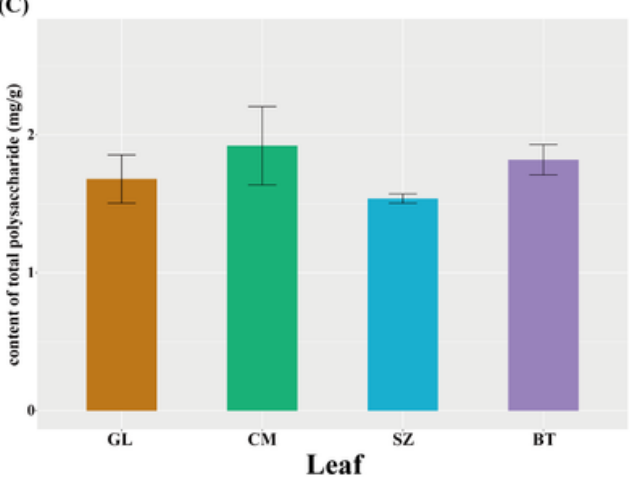

Figure 1

The total content of nine monosaccharide in the (A) root, (B) stem and (C) leaf of four ginseng cultivars.

*Significant at the 0.05 probability level. 
(A)

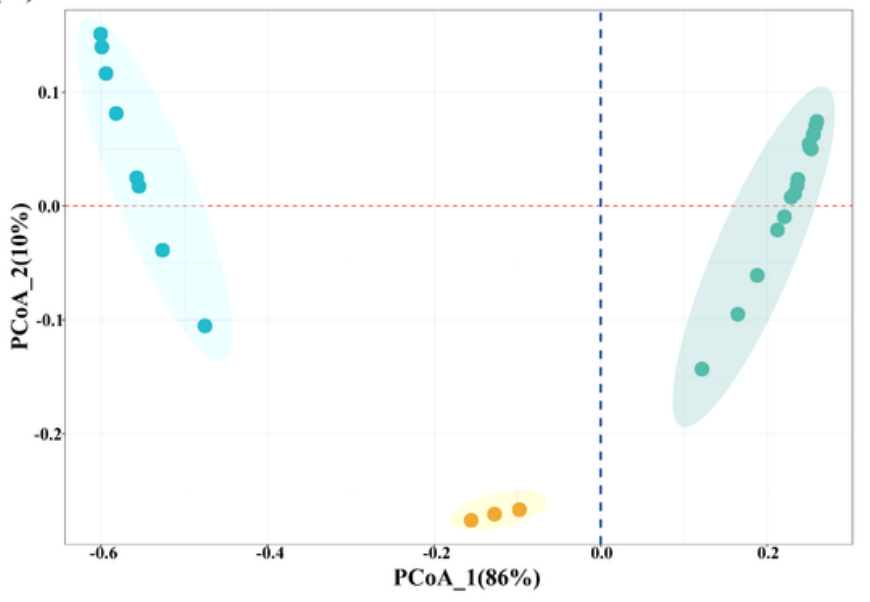

(B)

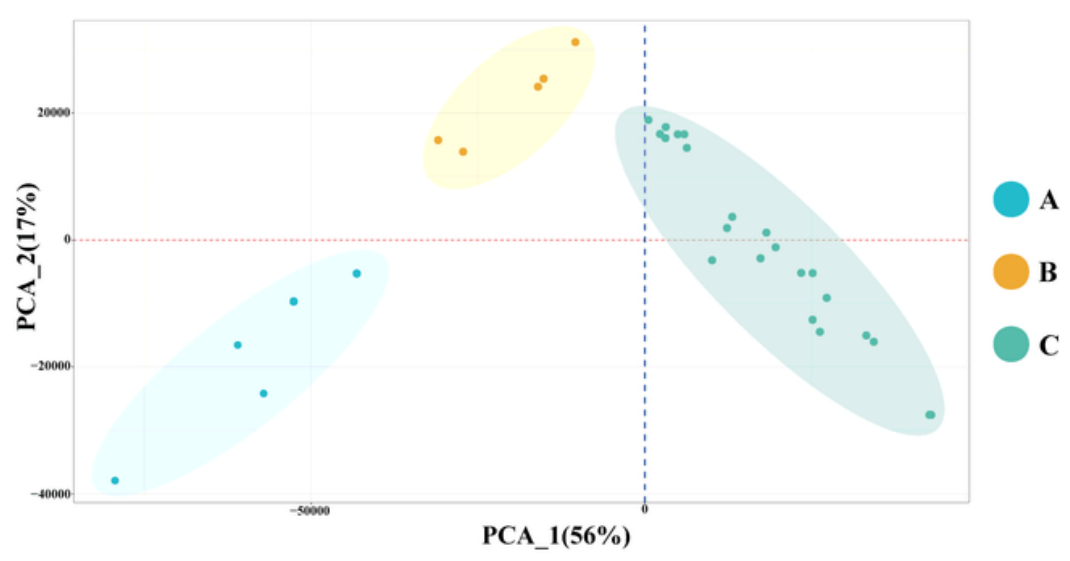

Figure 2

PCA score plot in monosaccharides content and transcriptome profiles of ginseng samples. (A), PCA score plot in nine monosaccharides content of four ginseng cultivars. Each point in PCA score plot representing an independent biological replicate. a, the roots sample from GL, CM and BT; b, the roots sample from SZ; c, the stems and leaves from GL, CM, BT and SZ. (B), PCA score plot in transcriptome profile of four ginseng cultivars. a, the roots sample from GL and $\mathrm{CM}$; $b$, the roots sample from BT and SZ; c, the stems and leaves from GL, CM, BT and SZ.

\section{Figure 3}

Kyoto Encyclopedia of Genes and Genomes (KEGG) pathway enrichment of differentially expressed genes (DEGs) in each of the comparison groups.

\section{Figure 4}

Putative pathway for polysaccharides biosynthesis in ginseng. The purple word represented enzyme involved in polysaccharides, and blue frames represented polysaccharides products. The red and blue blocks represent high and low expression levels, respectively. D-fructose-6p, D-fructose-6phosphate; D-man-6p, Dmannan-6phosphate; D-man-1p, D-mannan-1 phosphate; GDP-D-Man, GDP-D-mannose; GDP-4-oxo-6-deoxy-D-Man, GDP-4-oxo-6-deoxy-D-mannose; GDP- Fuc, GDP-L-fuc; D-glc-1p, D-glucose-1 phosphate; D-glc-6p, D-glucose-6phosphate; UDP-Glc: UDP-glucose; UDP-Gal, UDP-galactose; UDP-glcA, UDP-glucuronate; UDPD-xyl, UDP-D-xylose; UDP-L-Ara, UDP-L-arabinose; UDP-GalA, UDP-D-galacturonate; UDP-4-keto-6-deoxy-D-Glc, UDP-4-keto-6-deoxy-D-glucose; UDP-4-keto-Rha, UDP-4-keto-rhamnose; UDP-Rha, UDP-rhamnose; GAE, UDP-glucuronate 4-epimerase; GALE, UDP-glucose 4-epimerase; GMDS, GDP-mannose 4,6-dehydratase; GMPP, mannose-1-phosphate guanylyltransferase; GPI, glucose-6-phosphate isomerase; HK, hexokinase; MPI, mannose-6-phosphate isomerase; PGM, phosphoglucomutase; PMM, phosphomannomutase; RHM, UDP-glucose 4,6-dehydratase; sacA, beta-fructofuranosidase; scrK, fructokinase; SUS, sucrose synthase; TSTA3, GDP-L-fucose synthase; UXE, UDP-arabinose 4-epimerase; UGDH, UDP-glucose 6-dehydrogenase; UGP2, UTP-glucose-1-phosphate uridylyltransferase; UXE, UDP-arabinose 4-epimerase; UXS1, UDP-glucuronate decarboxylase. 
(A)

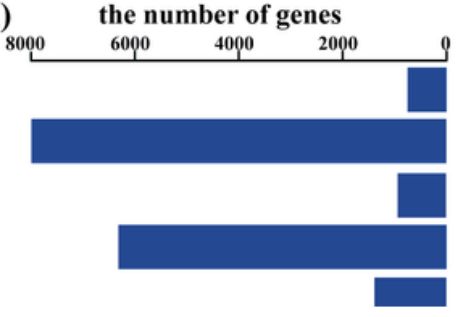

(B)

\begin{tabular}{|c|c|c|c|c|c|c|c|c|c|c|}
\hline $\tan$ & lasts & $\begin{array}{l}0.3 \\
0.0 .6 \\
(0.9)\end{array}$ & -0.027 & $\begin{array}{l}0.0027 \\
\text { (i) }\end{array}$ & $\begin{array}{l}-0.03 \\
(0.3) \\
(0.3)\end{array}$ & - & $\frac{-0.18}{(0.8)}$ & $\begin{array}{l}-0.37 \\
0.05\end{array}$ & $-\frac{0.66 .6}{(0.21)}$ & 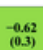 \\
\hline turquoise & $\frac{2.4}{(0.6)}$ & 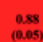 & $\begin{array}{l}0.06 \\
(0.2)\end{array}$ & $\begin{array}{l}0.67 \\
(0.2)\end{array}$ & $\begin{array}{l}\frac{1.013}{(0.8)} \\
(0.8)\end{array}$ & 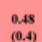 & 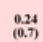 & $\begin{array}{l}0.057 \\
0.059)\end{array}$ & $\frac{-0.37}{(0.5)}$ & 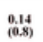 \\
\hline reenyellow & (2.9992) & $\begin{array}{l}.0 .63 \\
(0.3)\end{array}$ & 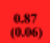 & $\begin{array}{l}0.055 \\
(0.057)\end{array}$ & 20.993 & $\begin{array}{l}0.07 \\
0.2 .2) \\
(0.2)\end{array}$ & 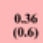 & (aAs) & 泪. & (2:203) \\
\hline blue & $\begin{array}{l}0.26 \\
0.2 .7 \\
0.9\end{array}$ & (0.9) & $\begin{array}{l}0.063) \\
(0.9)\end{array}$ & a.912 & $\begin{array}{l}2.7 \\
(022)\end{array}$ & 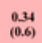 & 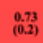 & 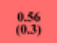 & 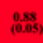 & 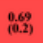 \\
\hline purpp & -0.28 & -2009 & -0.23 & -0.26 & 0.005 & 0.0988 & 2.6 & 0.2 & 0.8 & 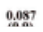 \\
\hline
\end{tabular}

\section{Figure 5}

Results of the gene co-expression network analysis based on WGCNA. (A), The number of genes contained in each module. (B), Correlation coefficient between polysaccharides and module eigengenes presented with a color scale with red and green representing positive and negative correlations, respectively. Glc, Gal, Rha, Man, Xyl, Ara, GlcA, GalA, Fuc and total represent glucose, galactose, rhamnose, mannose, xylose, arabinose, glucuronic acid, galacturonic acid, fucose and total polysaccharides, respectively. (C) and (D) represent KEGG annotation analysis of genes in the greenyellow and brown modules. The x-axis represents the gene ratio (annotation number/background number), and the y-axis represents the pathway name.

\section{Figure 6}

Construction of gene regulation network and correlation analysis. (A), Gene co-expression subnetwork of the greenyellow module. (b), Gene co-expression subnetwork of the brown module. (C), Correlation analysis of TFs and polysaccharides synthesis genes in the greenyellow module. (D), Correlation analysis of TFs and polysaccharides synthesis genes in the brown module. Network was reconstructed by edge weight cutoff $=0.50$ and visualized by Cytoscape. The red and blue blocks represent positive and negative correlations, respectively.

\section{Supplementary Files}

This is a list of supplementary files associated with this preprint. Click to download.

- Additionalfile1.docx

- Additionalfile2.docx

- Additionalfile3.docx

- Additionalfile4.docx 